\title{
A Systematic Review of the Orthopedic Literature Involving National Hockey League Players
}

This article was published in the following Dove Press journal:

Open Access Journal of Sports Medicine

\section{Emma R Berube (iD \\ Cesar D Lopez (D) \\ David P Trofa \\ Charles A Popkin (D)}

Center for Shoulder, Elbow and Sports Medicine, Department of Orthopedics, Columbia University Medical Center, New York, NY, USA
Correspondence: Charles A Popkin Center for Shoulder, Elbow and Sports Medicine, Department of Orthopedics, Columbia University Medical Center,

New York, NY, USA

Tel + I 2I2-305-4565

Email cp2654@columbia.edu
Background: Orthopedic injuries of National Hockey League (NHL) players are common and may significantly affect players' health and careers. Evidence-based injury management is important in guiding players' timely return to sport and their ability to play at their preinjury levels of competition.

Purpose: To summarize all data published between January 1980 and March 2020 on orthopedic injuries experienced by professional ice hockey players competing in the NHL.

Study Design: Systematic review.

Methods: A literature review of studies examining orthopedic injuries in the NHL was performed using the Embase, PubMed, and CINAHL databases. The review included studies focusing on NHL players and players attending the NHL Combine and preseason NHL team camps. Studies pertaining to non-orthopedic injuries and case reports were excluded.

Results: A total of 39 articles met the inclusion criteria and were analyzed. The articles were divided by anatomic site of injury for further analysis: hip and pelvis (24\%), general/other $(14 \%)$, ankle $(10 \%)$, knee $(10 \%)$, foot $(7 \%)$, shoulder $(7 \%)$, thigh $(7 \%)$, trunk $(7 \%)$, spine $(6 \%)$, elbow (4\%), and hand and wrist (4\%). The majority of articles were Level IV Evidence (51.3\%), followed by Level III Evidence (38.5\%). Most studies obtained data from publicly available internet resources $(24.7 \%)$, player medical records $(19.5 \%)$ or surveys of team physicians and athletic trainers (15.5\%). A much smaller number of studies utilized the NHL Injury Surveillance System (NHLISS) (6.5\%) or the Athlete Health Management System (AHMS) (2.6\%).

Conclusion: This systematic review provides NHL team physicians with a single source of the current literature regarding orthopedic injuries in NHL players. Most research was published on hip and pelvis (24\%) injuries, did not utilize the NHLISS and consisted of Level IV Evidence.

Keywords: ice hockey, National Hockey League, NHL, injuries, epidemiology

\section{Introduction}

Ice hockey is a highly skilled sport, a unique blend of speed, power and finesse. With vulcanized rubber pucks traveling at speeds approaching $100 \mathrm{mph}$, unforgiving fiberglass boards and numerous high-speed collisions, it is a sport with numerous intrinsic hazards. ${ }^{1}$ Not surprisingly, it has one of the highest injury rates in all of sports. ${ }^{2}$ Orthopedic injuries of National Hockey League (NHL) players are common and may significantly affect players' abilities to return to play and compete at the highest level. Due to the high-profile nature of the NHL, player injuries and team injury reports are closely followed by the media and often publicly reported, making it possible to track player injuries. As a result, there 
have been several published studies in the literature focusing on publicly available NHL player data in order to assess changes in player performance and return to play as a result of injuries. ${ }^{3-9}$ Additionally, surveys of NHL team physicians and ancillary medical staff provide valuable insights into player injuries, including common causes and recovery. Further studies have utilized the NHL Injury Surveillance System (NHLISS) and the Athlete Health Management System (AHMS), which are centralized, de-identified medical databases, developed to longitudinally track player medical histories. Injury surveillance systems (ISSs) are increasingly being employed by several professional and amateur sports leagues, including the NHL, and play a crucial role in objectively gathering information on sports injuries, including injury types, rates, mechanisms, risk factors, and anatomical sites. ${ }^{10-13}$ Such information helps guide effective injury prevention efforts, interventional assessments, focused treatment, and even changes in league rules and equipment, as seen in the National Football League's (NFL) response to player concussion data. ${ }^{14-18}$

The purpose of this systematic review is to summarize all data published between January 1980 and March 2020 on orthopedic injuries experienced by professional ice hockey players competing in the NHL. This review also aims to characterize the existing orthopedic literature based on anatomic injury site, data source, and level of evidence, while comparing results among different data sources, including the NHLISS and AHMS, medical records, team medical staff surveys, and other internetbased sources. Additionally, current literature will be stratified according to the location of injury, level of evidence and primary source of injury data, with comparisons made to identify differences observed between investigations using different data sources.

\section{Methods}

This systematic review was designed to follow the Preferred Reporting Items for Systematic Reviews and Meta-Analyses (PRISMA) guidelines. Three electronic databases - CINAHL, Embase and PubMed - were searched to identify all orthopedic literature involving NHL players. Boolean operators were used with "hockey," "hockey player," "ice hockey," "national hockey league," "professional hockey," "orthopedics," "orthopedic surgery," "orthopedic procedures," "orthopedic injury," "athletic injuries," and "wounds and injuries," as search terms to maximize the sensitivity of the search. To be included, articles needed to be original studies involving orthopedic injuries in NHL players. Studies involving hockey recruits attending the NHL Combine and hockey players attending the preseason NHL team camps were also included regardless of whether they played on the final team rosters. Exclusion criteria included literature review articles, studies with only an abstract, editorials, non-English language articles, case reports, non-ice hockey studies, studies of non-orthopedic injuries including concussions, facial, and vascular injuries, and non-NHL studies. Even if a study included NHL players, it was excluded if the NHL player data was grouped together with other player data such that it could not be analyzed on its own. No limits were placed on the level of evidence or timing of the studies. The search period was from January 1980 to March 2020. Articles were initially screened by title, then by abstract, and finally by full text. Publication year, source of data, study design, level of evidence, number of teams, study duration, total players, number of injuries, player demographic information, player position, recovery time, return to sport, player performance following return, injury site, type of injury, and treatment were extracted from the included articles. Articles were then separated into eleven categories based on injury site - spine, shoulder, elbow, hand and wrist, trunk, hip and pelvis, thigh, knee, ankle, foot, and general/ other - for further analysis of levels of evidence and sources of data, as per Kluczynski et al. ${ }^{19}$ Sources of data included the NHLISS, the AHMS, publicly available data from the internet, data from a single physician or institution, player medical records, surveys of team physicians or trainers, surveys of players, surveys of team equipment managers, and data gathered by trained scorers. Neither injury sites nor data sources were mutually exclusive, with some articles having multiple injury sites and data sources.

\section{Results}

A total of 2650 articles were identified from the search of the three databases. After 789 duplicates were removed, 1861 articles were screened for eligibility by title and abstract. 1634 articles were excluded according to inclusion and exclusion criteria. The remaining 227 articles underwent review of the full text, and 188 were excluded according to the same criteria. Thirty-nine were included in our systematic review, totaling 13,850 total players (Figure 1). The most common injury sites examined by the articles were hip and pelvis (18 articles), general/other 
(11 articles), ankle ( 8 articles), knee ( 8 articles), foot (5 articles), shoulder (5 articles), thigh (5 articles), trunk (5 articles), spine (5 articles), elbow (3 articles), and hand and wrist (3 articles) (Figure 2). Most articles were Level IV Evidence (51.3\%), followed by Level III Evidence (38.5\%), and Level II Evidence (7.7\%). One article was Level V Evidence (2.6\%) and no articles were Level I Evidence. Injury site was used to stratify the articles by level of evidence, as shown in Figure 3. The most common sources of data used by the articles were publicly available data on the internet (24.7\%), player medical records $(19.5 \%)$, surveys of team physicians or trainers (15.5\%), single physicians or institutions (14.3\%), surveys of players (14.3\%), the NHL Injury Surveillance System (6.5\%), the Athlete Health Management System (2.6\%), surveys of team equipment managers $(1.3 \%)$, and data gathered by trained scorers $(1.3 \%)$. Data sources were also compared by injury site, as shown in Table 1 .

\section{General Information}

Three of the four studies examining overall injury incidence reported injury incidences ranging from 13.6 to 17 injuries per 1000 athlete-exposures, with an athlete-exposure defined as participation in a game in McKay et al and as participation in a game or practice in Tyler et al. ${ }^{13,21,22}$ McKay et al defined an injury as an event sustained during a practice or game that caused the player to miss at least 1 game, while Tyler et al defined an injury as any event that caused the player to miss at least 1 game or practice or required attention of the team physician. ${ }^{13,21,22}$ Hutchinson et al reported a higher incidence of 119 injuries per 1000 athleteexposures, defining an injury as any event requiring evaluation or assistance from the medical team during a game, and not defining an athlete-exposure. ${ }^{13,21-23}$ Within a single season, $50.9 \%$ of all NHL players participating in regular season games missed at least 1 game due to injury, and over a 3 season period, $63.1 \%$ missed at least 1 game due to injury. ${ }^{24}$
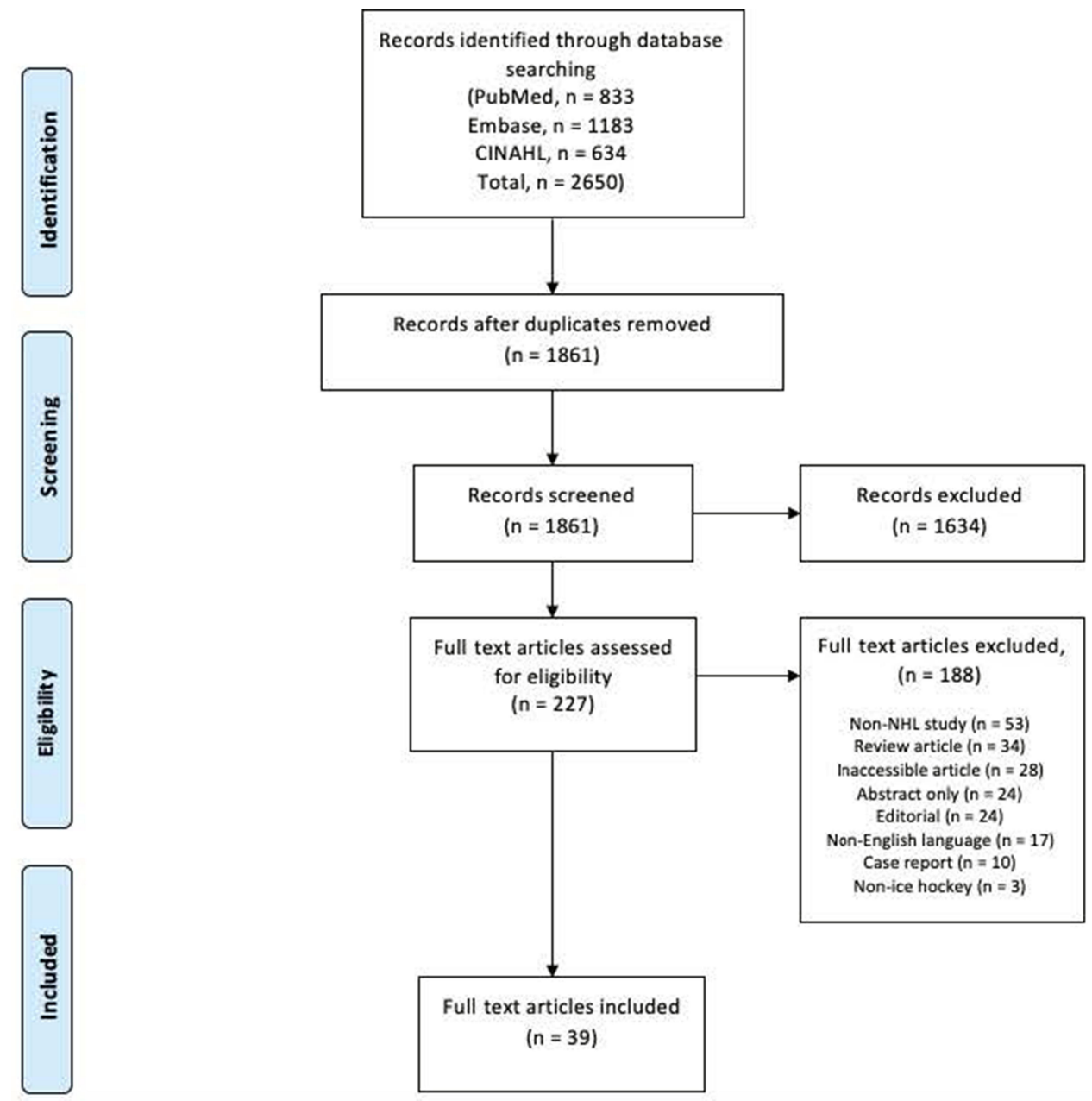

Figure I PRISMA (Preferred Reporting Items for Systematic Meta-Analyses) diagram. ${ }^{20}$ 


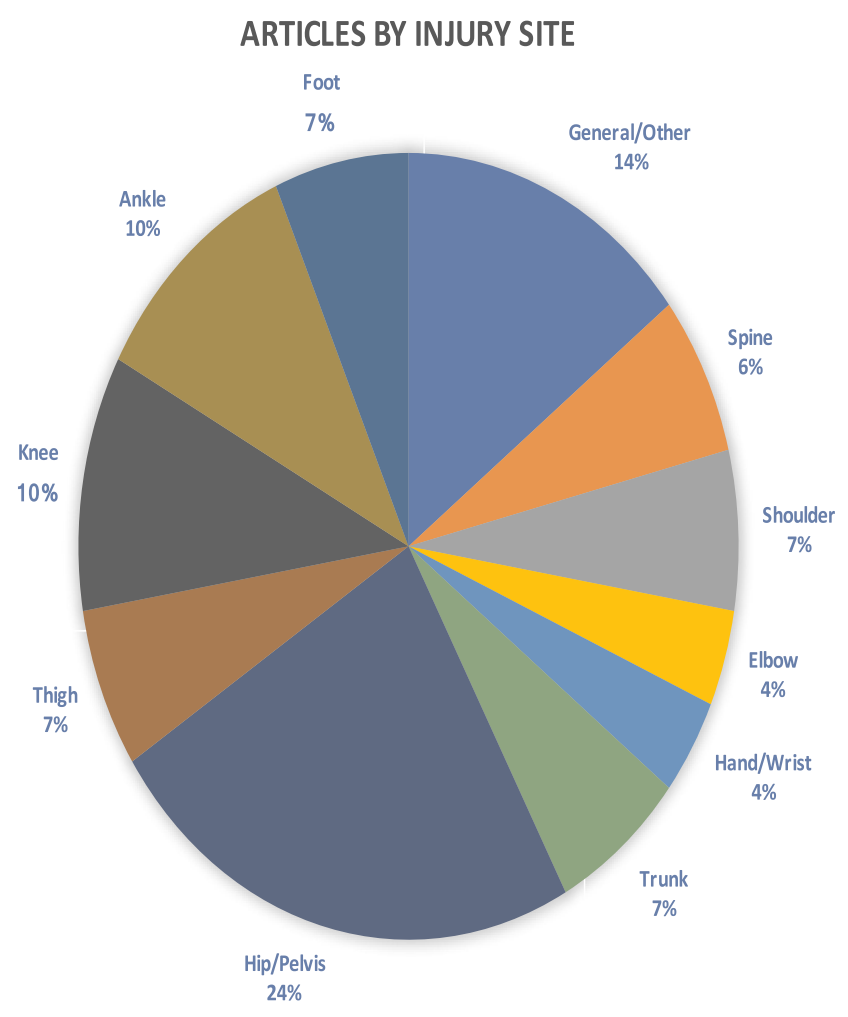

Figure 2 Percentage of articles by injury site of orthopedic injury. Injury sites are not mutually exclusive.
Several studies investigated when during the season and under what circumstances most injuries occurred. Injuries were more common during games than practice and during the regular season than the postseason. ${ }^{13,25-27}$ Some studies reported injuries were equally common throughout all game periods, while others reported greater occurrence of injuries in the first period than the second period or the third period. ${ }^{13,25-27}$ Injuries were also reported to be more common during home games than away games and during the end of the regular season compared to the beginning of the regular season. ${ }^{13}$

In examining overall injury, the most common mechanism of injury was body-checking, particularly receiving a body check. $^{13,23}$ Other common mechanisms of injury included incidental contact, being hit by a puck or stick, contact with the environment such as the boards or skate equipment, noncontact mechanisms, and fighting. ${ }^{13,23}$ McKay et al additionally assessed mechanism of injury by man-games lost, and found that receiving a body check was responsible for most man-games lost, followed by incidental contact and being hit by a puck. ${ }^{13}$

\section{ARTICLES BY LEVEL OF EVIDENCE AND INJURY SITE}

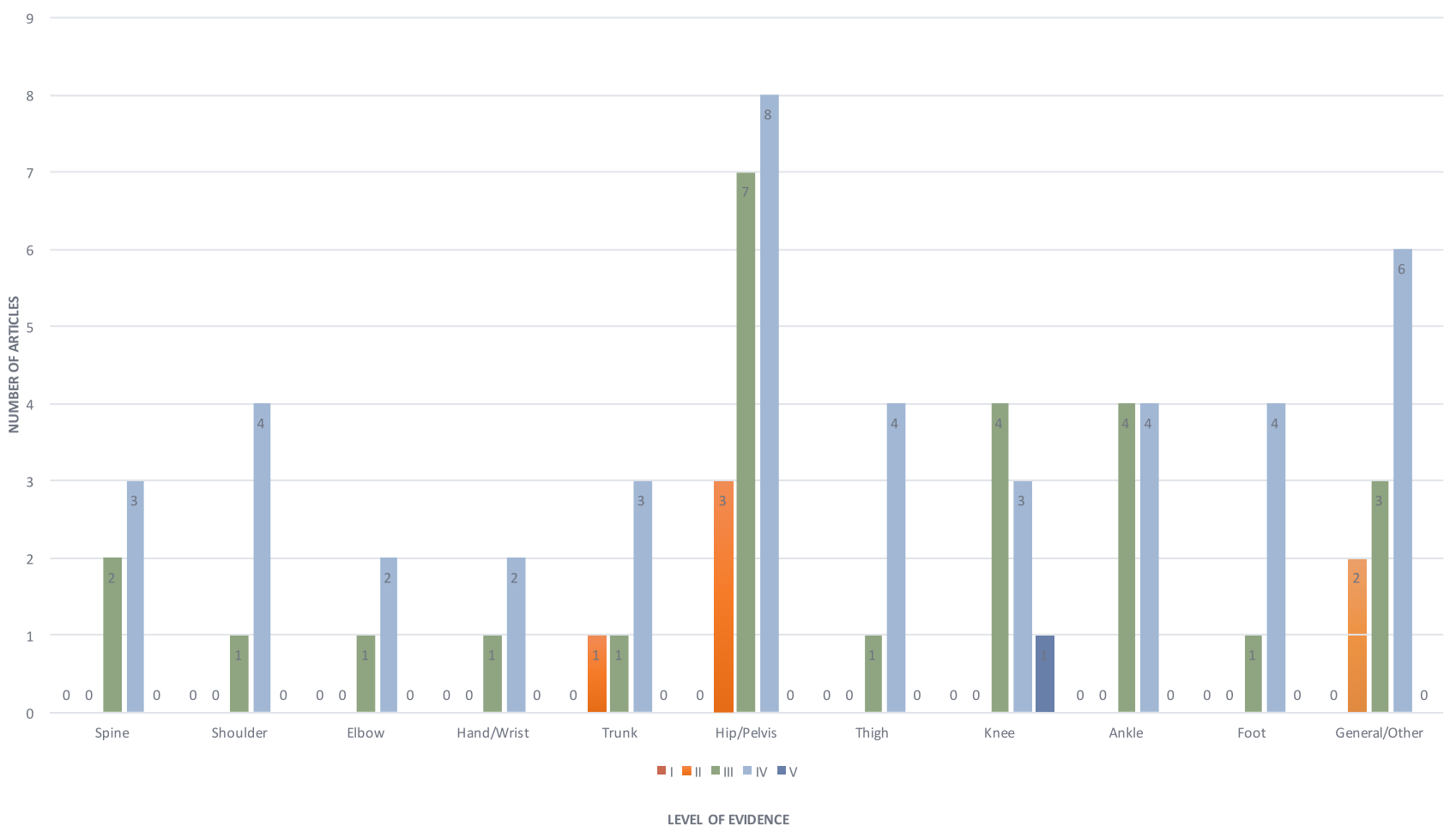

Figure 3 Number of articles by level of evidence and injury site of orthopedic injury. Injury sites are not mutually exclusive. There were no Level I studies. 


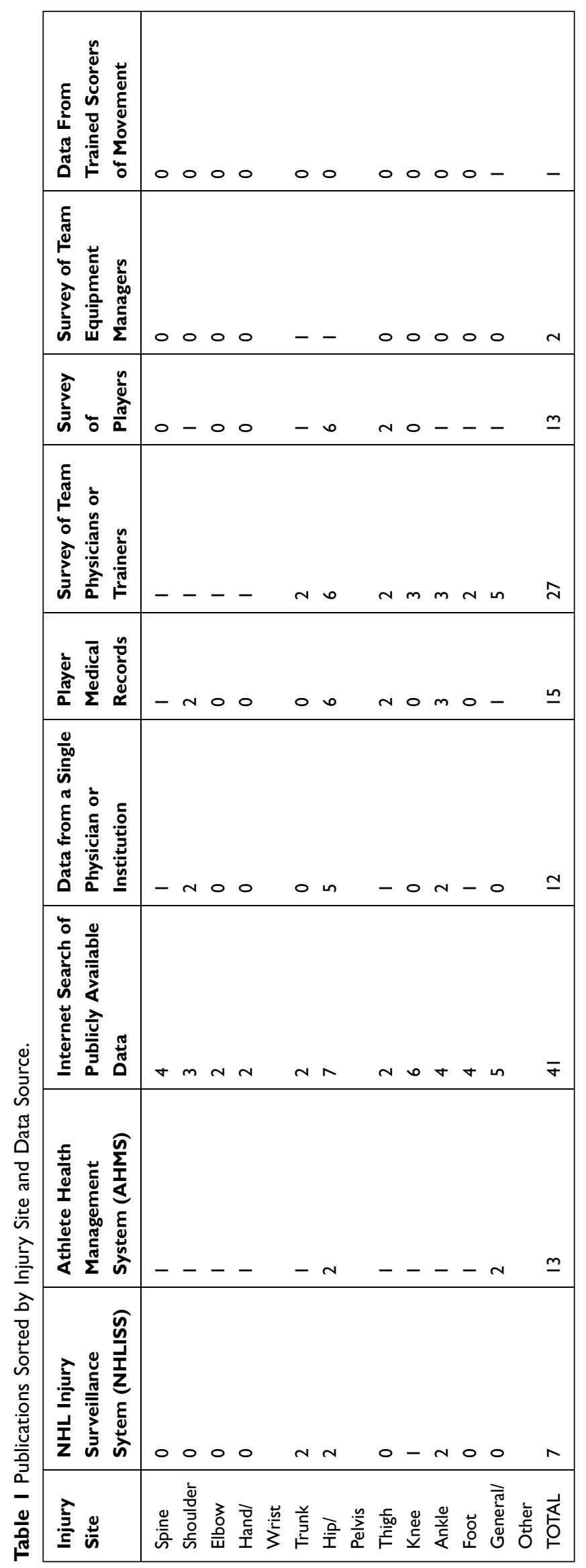


McKay et al was the only study to assess the relationship between all types of orthopedic injury and player position, and found that defensemen were more likely to report a game-related time-loss injury than forwards. ${ }^{13}$ Goalies appeared to suffer more hip injuries compared to forwards and defensemen, but did not differ in their capacities to return to play and their prior level of performance following surgery. ${ }^{5,6,26,28}$ Forwards appeared to suffer more ankle syndesmosis sprains than defensemen or goalies, but there was no difference in time lost from play. ${ }^{27,29}$ Forwards were also affected by more groin injuries than players of other positions, and had the largest decrease in time played and in performance following ACL reconstruction compared to players of other positions. ${ }^{25,30,31}$

\section{Spinal Injuries}

The literature regarding orthopedic spinal injuries in NHL players focused on cervical and lumbar spine injuries. While no study examined the frequency of isolated spinal injury, over a period of 6 seasons McKay et al reported that $12 \%$ of total injuries were injuries of the neck, chest/ ribs/upper back, and pelvis/lower back. ${ }^{13}$

\section{Cervical Disk Herniation}

Because of a paucity of information in the literature regarding outcomes following surgical intervention for cervical disk herniation in high-level athletes, recent studies have sought to define such outcomes. A review of professional athletes undergoing 27 anterior cervical discectomy and fusion (ACDF) procedures included 5 procedures in NHL athletes. ${ }^{32} \mathrm{NHL}$ athletes returned to play at a rate of $100 \%$ in this study. Though radiographic evidence of a fusion was a requirement for return to play, one NHL athlete in this study returned at 6.7 months, despite imaging showing lack of bony fusion. ${ }^{32}$ He played 4 additional seasons symptom free before sustaining an acute injury at which time a CT Scan showed a nonunion at the surgical level. He underwent a revision with posterior instrumentation and fusion and returned at 7.8 months. ${ }^{32}$ A study examining the difference in outcomes between the different surgical procedures for cervical disk herniation ACDF, posterior foraminotomy (PF), and total disk replacement (TDR) - included 101 professional athletes, 5 of whom were NHL players. ${ }^{4}$ Four underwent ACDF and 1 underwent PF. All NHL players in this study were able to return to sport.

\section{Lumbar Disk Herniation}

Schroeder et al examined outcomes for operative and nonoperative treatment of lumbar disk herniation in NHL players, as previous studies had neither focused specifically on hockey players nor demonstrated a consensus on whether athletes undergoing lumbar fusion could return to play. ${ }^{33}$ Of 87 NHL players diagnosed with symptomatic lumbar disk herniation, 31 were managed nonoperatively while 56 were managed surgically - 48 with discectomy and 8 with single-level fusion. The return to play (RTP) rate in NHL Players was $85 \%$ overall but with decreased on-ice performance. There was no significant difference noted in outcomes between the nonoperative and operative groups. The authors concluded that lumbar fusion is compatible with return to play, with high RTP rates regardless of treatment type. ${ }^{33}$

\section{Shoulder}

Shoulder injuries accounted for $10-12 \%$ of total injuries (including head and neck injuries) to NHL players. ${ }^{13,23,24}$ For each shoulder injury, players missed an average of 10.2 games. $^{24}$

Because of the aggressive and high-impact nature of ice hockey, patterns of shoulder injury in hockey players may differ from those of the general population. Using Magnetic Resonance Imaging (MRI)/Magnetic Resonance Angiography (MRA), Dwyer et al characterized injury patterns in professional ice hockey players who presented following an episode of traumatic shoulder instability. ${ }^{34}$ Bankart (anteroinferior glenoid labrum) lesions were the most common lesions identified (14 NHL players), followed by Hill-Sachs (posterolateral humeral head) lesions (10 NHL players), other lesions (5 NHL players), superior labrum anterior and posterior (SLAP) lesions (3 NHL players), and posterior labral lesions (3 NHL players). Most (79\%) athletes with Bankart lesions also had HillsSachs lesions. When analyzed by position, defensemen were found to have the lowest prevalence of Bankart lesions. $^{34}$

Rangavajjula et al focused specifically on labral tears, performing an assessment of outcomes following arthroscopic labral repair, as time to return to play and postoperative performance had not been previously examined. ${ }^{35}$ Eleven NHL players were included who had a total of 13 labral injuries. Eight of the 13 injuries were on the player's dominant shoulder. Eight of these injuries were acute -4 due to contact with another 
player and 4 due to contact with the boards - and 5 were chronic occurring over several seasons of play. All players returned to play, at an average of 4.3 months, with no difference in time to return to play between dominant shoulder and nondominant shoulder injuries, between offensive and defensive players, or between acute and chronic injuries. There was no difference in on-ice performance. ${ }^{35}$

\section{Elbow}

No articles included in this systematic review focused specifically on elbow injuries. Elbow injuries were responsible for $2 \%$ of total injuries in NHL players. ${ }^{13,23}$ Hand and arm injuries accounted for a greater portion of injuries $(8.6 \%)$, and were responsible for 10.3 missed games on average per injury, second only to missed games from head/neck injuries. ${ }^{24}$

\section{Hand and Wrist}

No articles included in this systematic review focused specifically on hand and wrist injuries. Wrist injuries accounted for $1-2 \%$ of total injuries in NHL players and hand injuries accounted for $3-7 \%$ of total injuries. ${ }^{13,23}$

\section{Trunk}

Injuries of the chest and abdomen made up $7.6 \%$ of total injuries in NHL players. ${ }^{24}$ In the literature, trunk injuries are often grouped with groin injuries, so distinguishing findings about trunk injuries alone can be difficult. Using a retrospective review of all $7050 \mathrm{NHL}$ players from the 1991-1992 season through the 1996-1997 season, Emery et al investigated groin and abdominal strains in NHL players. ${ }^{25}$ Over the 6 season period, 617 groin and abdominal strains occurred $-76.8 \%$ groin strains and $23.2 \%$ abdominal strains. All players returned to play, missing on average 10.6 practices or games for abdominal injuries, and 6.6 practices or games for groin injuries. Almost one quarter of these injuries were recurrent. The majority occurred through non-contact mechanisms, differentiating them from most other types of injuries in hockey which occur primarily by contact. Offensive players were most affected by groin and abdominal injuries $(60.6 \%$ of groin and abdominal injuries), followed by defensive players $(28.9 \%)$, and goalies $(5.5 \%)$. The incidence of groin and abdominal injuries increased over the 6 seasons studied, from 12.99/100 players/year in 1991 to 19.87 injuries/100 players/year in $1996 .^{25}$
After defining the problem of groin and abdominal injuries in NHL players, Emery et al prospectively examined intrinsic and potentially modifiable risk factors for injury, as there previously had been no literature focusing on risk factors for groin and abdominal strains in hockey players. $^{30}$ Using a population of 1292 NHL players attending preseason training camps for the 1998-1999 season, the authors reported 52 injuries during training camp and 152 during the regular season, consistent with the rates previously reported. ${ }^{25,30}$ This study additionally broke down reinjury rates by type of strain, and found a reinjury rate of $33.3 \%$ for abdominal strains and $13.3 \%$ for groin strains. ${ }^{30}$ During training camp, risk factors for groin and abdominal injury were less sports-specific training, previous injury, and veteran status (at least 1 year of NHL experience). In the regular season, only veteran status was a significant risk factor. Peak isometric adductor torque, total abduction flexibility, and skate blade hollow measurements were not significant risk factors during training camp or the regular season. The authors identified veterans as the population that would most benefit from more off-season sport-specific training. ${ }^{30}$

\section{Hip and Pelvis}

Hip and pelvis were the most frequently encountered injury sites in this systematic review yet only made up approximately $10 \%$ of total injuries in NHL players in previous studies. ${ }^{13,23}$ In addition to being grouped with trunk injuries, groin injuries are often instead discussed with hip/pelvis injuries complicating the definition of a groin injury and its relationship to trunk and abdominal injuries.

Rather than focusing on a specific pathology, Epstein et al sought to characterize the range of intra-articular hip injuries suffered by NHL players and determine their relationships to player positions. ${ }^{26}$ Over 4 seasons, 1441 NHL players suffered 890 hip and groin injuries, 94 of which were intra-articular (10.6\% of all hip and groin injuries). Hip labral tears were the most frequently encountered intra-articular hip injury (69.1\%). Compared to players with all other hip and groin injuries, those with intra-articular injuries were older, missed more games, and missed more days of play. Compared to forwards and defensemen, goalies had a relative risk of 4.78 injuries per 1000 player-game appearances. ${ }^{26}$

Christian et al and Schallmo et al also looked at a variety of hip and pelvic pathologies in NHL players 
(labral tears, femoracetabular impingement, chondral defects and loose bodies), in the context of other professional sports leagues. ${ }^{5,9}$ NHL Players $(n=41)$ underwent 49 hip arthroscopies in the Christian study and had the highest return to play of all professional sports leagues at $95.9 \%{ }^{9}$ There were 55 NHL players that underwent 73 hip arthroscopies in the Schallmo study with a return to play of $91.7 \% .^{5}$ The breakdown in return by position was $36 / 38$ forwards (94.7\%), 13/14 defensemen (92.9\%) and $18 / 21$ goalies $(85.7 \%) .^{5}$ Those who returned to play played significantly more games in the prior season than those who did not return to play. ${ }^{5}$ Players undergoing bilateral hip arthroscopy returned to play at rates similar to those undergoing unilateral, while players undergoing revision arthroscopy returned at lower rates than those undergoing primary arthroscopy. NHL players in both studies returned to play sooner than athletes in the other professional sports leagues. 5,9 More games played during the prior season were predictive of shorter recovery time and a longer postoperative career length, while player age was a negative predictor of postoperative career length. ${ }^{5}$ NHL player performance decreased significantly in the first season postoperatively, but recovered in Schallmo et al over the second and third seasons. ${ }^{5,9}$ This decrease in performance was unique to NHL players in both studies. ${ }^{5,9}$ Schallmo et al additionally analyzed RTP rates and performance outcomes by position, and found that unlike forwards and defensemen, goalies did not have significant differences in RTP rates compared to other sports nor significant performance decreases. ${ }^{5}$

Femoroacetabular impingement (FAI) is common in NHL players. $^{36}$ In a group of 130 athletes undergoing preseason entrance exams for the 2014-2015 NHL season, only $32(24.6 \%)$ reported a history of hip pain and none had undergone previous hip surgery. ${ }^{28}$ However, radiography demonstrated cam-type FAI in $69.4 \%$ of hips, suggesting there are a significant number of elite hockey players with asymptomatic FAI. Goaltenders had the highest prevalence of FAI on radiography $(93.8 \%) .{ }^{28}$ Larson et al also found a high prevalence of asymptomatic FAI in NHL players in a study seeking to characterize radiographic hip anatomy in NHL players and correlate it with hip range of motion, symptoms, and surgery. ${ }^{36}$ On range of motion examination, greater cam-type morphology was correlated with decreased hip internal and external rotation at $90^{\circ}$ of flexion and straight and butterfly abduction, while a positive crossover sign was correlated with increased hip abduction. Decreased hip external rotation and total arc of motion were predictors of hip-related pain and hip surgery. ${ }^{36}$

Both Menge et al and Philippon et al investigated outcomes in NHL players following arthroscopic hip surgery for symptomatic FAI. ${ }^{6,37}$ All hips ( $n=28$ hips) included in Philippon et al demonstrated radiographic cam-type FAI and $85 \%$ demonstrated pincer-type FAI. ${ }^{37}$ Of 69 hips included in Menge et al, most demonstrated radiographic mixed-type FAI with both cam and pincer deformities (74\%), followed by cam-predominant FAI (19\%) and pincer-predominant FAI (7\%). ${ }^{6}$ Intraoperatively, all hips in both studies were found to have labral lesions. ${ }^{5,36}$ Depending on the specific lesion, players underwent labral repair, and in some cases labral reconstruction with iliotibial band (ITB) autograft, osteoplasty, rim trimming, microfracture and debridement. ${ }^{6,37}$ The type of treatment did not affect postoperative career length. ${ }^{6}$

RTP rate was $100 \%$ in both studies, with an average time to return to skating and drills of 3.8 months. ${ }^{6,37}$ Decreased time from injury to surgery correlated with decreased time to return to play, and time from injury to surgery was increased in players with chondral defects. ${ }^{37}$ Players had an average Modified Harris Hip Score (MHHS) of 75 preoperatively, which improved to 95 postoperatively, though neither score was correlated with recovery time. Player age did not have a significant impact on recovery time. ${ }^{37}$ Mean career length after hip arthroscopy was 5.9 years. ${ }^{6}$ Longer career length after surgery was associated with younger age at the time of surgery, and shorter preoperative duration of symptoms. ${ }^{6,37}$ Goaltenders did not differ in their postoperative career length compared to other player positions. ${ }^{6}$

\section{Hockey Groin Syndrome}

Sports hernia, hockey groin syndrome, and athletic pubalgia are all terms used in the literature to refer to a tear in the abdominal external oblique muscle that causes lower abdominal and groin pain. ${ }^{7}$ Four studies focused on characterizing these injuries in terms of clinical and surgical findings, and assessing player outcomes following surgical repair. $^{7,38-40}$ Tears in the external oblique were found to be mainly central, with entrapment of the ilioinguinal nerve or its branches in the scar tissue of the torn areas. ${ }^{38-40}$ The abdominal internal oblique muscles were found to be significantly hypertrophied. ${ }^{39}$ On pathologic examination, the ilioinguinal nerve showed significant nerve injury with perineural fibrosis and axonal degeneration in greater than $20 \%$ of cases. ${ }^{38,39}$ Lacroix et al additionally noted 
lateral tears of the superficial inguinal ring with the conjoint tendon remaining intact. ${ }^{40}$ Recurrence occurred in $4-9 \%$ of cases, and all recurrences but one were managed operatively. ${ }^{38-40}$

RTP rates ranged from $91 \%$ to $100 \%{ }^{7,38-40}$ Postoperative performance decreased across all studies, with some players unable to return to their previous level of competition and instead returning to a lower level such as the American Hockey League (AHL) ${ }^{38,39}$ Preoperative playing time was negatively associated with postoperative performance; NHL players who had played greater than or equal to 7 seasons preoperatively hadsignificant decreases in games played, goals, assists, and time on ice compared to both controls and players who had played 6 or fewer seasons preoperatively. ${ }^{7}$

\section{Adductor Strain}

Adductor strains accounted for $68.3 \%$ of groin and abdominal injuries and $8 \%$ of all injuries to NHL players. ${ }^{22,25}$ Tyler et al studied 47 NHL players of a single team over 2 years to determine whether hip muscle strength and flexibility were risk factors for the development of adductor and hip flexor muscle strains. ${ }^{22}$ Eight players suffered 11 adductor strains, an incidence of 3.2 strains per 1000 player-game exposures. Forty-four percent of strains were recurrent and all players returned to play. Preseason hip strength testing successfully identified players at risk of developing hip adductor strains. Hip adduction-toabduction strength ratio was the best predictor of future adductor strain - a player was 17 times more likely to sustain an adductor strain if his adductor strength was less than $80 \%$ of his abductor strength. There was no difference in preseason hip flexibility between injured and uninjured players. ${ }^{22}$

To reduce the incidence of adductor strains, Tyler et al developed and implemented a preseason exercise program aimed at functional strengthening of the adductor muscles, consisting of 3 sessions per week for 6 weeks. ${ }^{21}$ Players were included in the intervention group if their adductor-to -abductor strength ratio was less than $80 \%$ - 33 players met this criteria out of a potential 58 tested. Over the following 2 years, 3 players suffered adductor strains, for an incidence of 0.71 adductor strains per 1000 playergames, significantly lower than that before the intervention. ${ }^{21,22}$ Overall injury incidence did not change and adductor muscle strains accounted for $2 \%$ of all injuries. ${ }^{21,22}$ None of the adductor strains were recurrent, though other players in the intervention group had histories of adductor strains. ${ }^{21}$ Seven players in the intervention group were retested the following year and demonstrated significant improvement in adduction-toabduction muscle strength ratios, further demonstrating the success of the exercise program. ${ }^{21}$

\section{Thigh}

Thigh injuries accounted for $3-14 \%$ of total injuries to NHL players. ${ }^{13,23}$ Contusions, femur fractures and hamstring injuries were identified in the systematic review. ${ }^{41}$

\section{Femur Fracture}

Using a case series of 4 professional athletes, 2 of whom were NHL players, Sikka et al investigated outcomes following treatment of femur fractures, as no studies had previously assessed return to play in this population. ${ }^{42}$ Both NHL players were injured when checked from behind, causing them to crash into the boards and sustain an axial load on the injured leg. They subsequently underwent anterograde intramedullary nailing with nonoperative management of a concomitant patellar fracture in one player. NHL players returned at an average of 7 months postoperatively and performed at or above their preinjury levels. $^{42}$

\section{Knee}

The majority of the literature in this systematic review involving knee injuries focused on ACL tears. Knee injuries accounted for $9-13 \%$ of total injuries to NHL players. ${ }^{13,23}$

\section{ACL Tear}

Over 13 years, Erickson et al identified 40 ACL tears in NHL players, for an approximate incidence of $3 \mathrm{ACL}$ tears per year in the NHL. ${ }^{8}$ They often occurred in conjunction with other injuries - in a series of 47 players Sikka et al found that $68 \%$ had concomitant meniscal injury and $68 \%$ had concomitant MCL injury. ${ }^{31}$ Most ACL tears occurred from contact with another player, or from sliding into the boards. ${ }^{31}$ Different surgical techniques exist for reconstructive ACL surgery in elite athletes. In a survey of NHL team orthopedic surgeons, autograft, specifically bone-patellar tendon-bone, was the most popular graft choice for ACL reconstruction in NHL players. ${ }^{43}$ Most of these surgeons also preferred a transtibial femoral tunnel drilling technique as opposed to an anteromedial or 2-incision femoral tunnel drilling technique, and all preferred a single bundle technique as 
opposed to a double bundle technique. The most popular RTP criteria for NHL surgeons were tests (Vail and single-leg hop), function (no pain, normal ROM, full strength, subjective stability), and greater than 6 months elapsed from time of surgery. Most did not recommend the use of a knee brace during play following return to play. ${ }^{43}$ Sikka et al found that the type of graft - patellar tendon autograft, hamstring autograft, bone-patellar tendon-bone allograft, or tibialis posterior allograft - had no impact on postoperative performance outcomes or complication rates. ${ }^{31}$ Reoperation rates varied from $2 \%$ to $20 \%$. $^{3,8,31}$

Return to play rates to the NHL following ACL reconstruction ranged from $89.4 \%$ to $97 \%$, with recovery times of 7.8 to 9.8 months. ${ }^{3,8,31}$ Postoperative career lengths varied from 2.8 years post-injury (which was less than the control group's 4.4 years) to 4.5 years postoperatively. ${ }^{3,8,31}$ While there was no relationship between severity of MCL injury and recovery time or career length, meniscal injury was associated with decreased career length compared to controls and those with isolated ACL injuries. ${ }^{31}$ Overall, there was no decrease in performance for players who returned, though when analyzed by player position forwards demonstrated decreased performance compared to their preinjury performance and controls. ${ }^{8,31}$ Mai et al compared the outcomes of NHL players with those of Major League Baseball (MLB), NFL, and National Basketball Association (NBA) players following ACL reconstruction and found that NHL players had the best prognosis with the highest rates of RTP, shortest recovery times, highest survival rates, longest career lengths, and no significant changes in performance (versus significant performance changes in other sports). ${ }^{3}$

\section{Chondral Defects}

While previous studies had investigated outcomes in professional athletes following microfracture, no study had included NHL players. To fill this gap, Schallmo et al investigated outcomes in 131 professional athletes (MLB, NBA, NFL), 3 of whom were NHL players, following primary microfracture of the knee. ${ }^{44}$ All NHL players returned to play. Average postoperative recovery time was 258 days and mean adjusted career length postoperatively was 2.9 years for NHL players, neither of which were significantly different from players of other sports. ${ }^{44}$

\section{Ankle}

Ankle sprains, boot-top tendon lacerations, and bone injuries were the ankle injuries discussed in the literature of this systematic review. Ankle injuries made up 1-6\% of total injuries to NHL players. ${ }^{13,23}$

\section{Boot-Top Tendon Lacerations}

"Boot-top" injuries include injuries to the anterior tibial tendon, extensor hallucis longus tendon, extensor digitorum longus tendons, or the dorsalis pedis artery, vein, or nerve. ${ }^{45}$ Because these injuries occur infrequently, there is little in the literature regarding their presentation and management. Simonet et al reported a case series of 5 players treated over a period of 5 years, 3 of whom were NHL players. ${ }^{45}$ Notably, all players were wearing their skate tongues down when the injuries occurred and the mechanism of injury was collision with another player. In 2 out of the 5 cases ( 1 out of 3 NHL players), the initial diagnosis was missed due to the lack of apparent seriousness of the skin laceration, delaying surgical treatment. All players underwent reconstructive surgery, and the NHL players were able to return to play with no long-term disability. ${ }^{45}$

\section{Ankle Sprains}

Though low (lateral) ankle sprains - injuries to the anterior talofibular ligament (ATFL), calcaneofibular ligament (CFL), and posterior talofibular ligament (PTFL) - are far more common than high ankle (syndesmosis) sprains injuries to the anterior-inferior tibiofibular ligament (AITFL), posterior-inferior tibiofibular ligament (PITFL) and interosseous ligament - in the general population and in athletes, that may not be the case in hockey players. ${ }^{27}$ A retrospective review of 2 NHL teams from 1991 to 2001 by Wright et al revealed that of the 19 ankle sprains sustained, 14 (74\%) were syndesmosis sprains. ${ }^{29}$ To assess the relationship between MRI findings and player outcomes following ankle syndesmosis sprain in NHL players, Mollon et al analyzed 105 NHL players, 20 of whom had MRIs available for review. ${ }^{27}$ The most common MRI findings were injury to the AITFL (95\% of MRIs), contusions ( $71 \%$ ), injury to the ATFL (52\%), and injury to the PITFL (43\%). ${ }^{27}$ Almost all syndesmosis sprains occurred from external rotation during games, particularly in the first period during the regular season. ${ }^{27,29}$ Forwards were most affected, followed by defenseman and then goaltenders. ${ }^{27}$ The mechanism of injury for the lateral 
sprains was loss of the skate edge causing inversion. ${ }^{29}$ All players with lateral sprains and all players but one with syndesmosis sprains in Wright et al were treated nonoperatively. ${ }^{29}$

All athletes in both studies returned to play, with an average recovery time of 45 days for syndesmosis sprains and 1.4 days for lateral sprains. ${ }^{27,29}$ Neither MRI findings nor player position correlated with return to play or recovery time in players with syndesmosis sprains. ${ }^{27,29}$ Following return to play, players with syndesmosis sprains reported several weeks of soreness and stiffness, and did not feel they had returned to normal until the following season. ${ }^{29}$ Career length following injury was unchanged, with the exception of the single reinjured player who reported that he felt his career had been shortened by the injury. ${ }^{29}$

\section{Foot}

Foot injuries made up 3-6\% of total injuries in NHL hockey players. ${ }^{13,23}$ While radiographs are commonly used as an initial diagnostic modality, not all severe bone contusions and fractures are visible. ${ }^{46}$ Baker et al investigated the occurrence, severity, and distribution of radiographically occult bone injuries occurring in 27 elite hockey players, 26 of whom were NHL players, using MRI. ${ }^{46}$ Twenty-four bone injuries were documented on the medial side of the foot and ankle, 13 in the central region, and 8 on the lateral side. Severe injuries, defined as fractures and high-grade contusions, were significantly more common in the medial bones (13 injuries) compared to the lateral or central bones ( 3 injuries). Of the 16 severe injuries, the most commonly affected bones were the tarsal navicular bone, followed by the medial malleolus, distal fibula, and first metatarsal base. The most common mechanism of injury was a direct blow from a moving puck, which compared with other mechanisms of injury was more likely to cause a severe injury, injure the medial bones, and impact defensemen. All players were initially managed nonoperatively, though 5 NHL players did not improve with nonoperative management and subsequent radiographs showed visible fractures. Four of these fractures were medial malleolar fractures for which the players underwent open reduction and internal fixation, and one was a high ankle sprain with a distal fibular fracture that was managed nonoperatively. An average of 10.6 games were missed for severe injuries, and 2.4 games were missed for non-severe injuries. $^{46}$
Citing a lack of outcomes in the literature regarding elite athletes undergoing operative management of foot fractures, Singh et al investigated RTP rates and postoperative performance in NBA, NFL, MLB, and NHL players. ${ }^{47}$ Of the 84 operative procedures, 3 occurred in NHL players. NHL players had first metatarsal fractures and a navicular fracture. NHL players were omitted from the performance analysis due to the small sample size, and the authors concluded that overall, athletes had excellent postoperative outcomes with a RTP of $98.7 \%$ regardless of sport and fracture location. ${ }^{47}$

\section{Comparison of Overlapping Findings from NHLISS and AHMS vs Medical Records vs Internet-Based Studies as Data Sources Hip Arthroscopy and Femoroacetabular Impingement}

Both Christian et al and Schallmo et al used internet-based sources - including injury reports, player profiles and biographies, press releases, and newspaper archives - to investigate outcomes for professional athletes following hip arthroscopy. ${ }^{5,9}$ Christian et al included 131 athletes from 2000 to 2016, 41 of whom were NHL players undergoing 49 arthroscopy procedures, and Schallmo et al included 180 athletes from 1999 to 2016, 55 of whom were NHL players undergoing 73 arthroscopy procedures. RTP rates and recovery times were similar between the 2 studies (with Christian et al reporting 95.5\% RTP rate and 164 days to RTP, and Schallmo et al reporting 91.8\% RTP rate and 177 days to RTP) but postoperative career lengths differed (with Christian et al reporting 0.9 seasons and Schallmo et al reporting 2.7 years). Both reported decreased performance during the first postoperative season, but this normalized in Schallmo et al by the second and third postoperative seasons. When compared to other sports Christian et al found worse performance, but no difference in RTP rates, recovery time, or postoperative career length, while Schallmo found worse performance, higher RTP rates, shorter recovery time, and shorter postoperative career length for NHL players compared to athletes of other sports. ${ }^{5,9}$ The discrepancy in findings between these 2 studies with similar study populations, time periods, and data sources suggests that medical information on professional athletes, including NHL players, available on the internet is likely incomplete.

Larson et al, Lerebours et al, Menge et al, and Philippon et al all investigated FAI in NHL 
players. ${ }^{6,28,36,37}$ Both Larson et al and Lerebours et al investigated radiographic hip anatomy in NHL players during preseason camps with Larson et al using player medical records to investigate player history and Lerebours et al relying solely on player surveys for history. $^{28,36}$ Larson et al included 59 players and Lerebours et al included 130 players. Despite their different data sources, both studies found a high prevalence of radiographic FAI (greater than 85\% cam-morphology in Larson et al, 69.4\% cam-type deformity in Lerebours et al), often occurring in asymptomatic players (31\% with history of hip pain in Larson et al, 24.6\% with history of hip pain in Lerebours et al). ${ }^{28,36}$ Menge et al and Philippon et al investigated outcomes following surgical intervention for FAI, with Menge et al using player medical records for operative information and the internet for career information and Philippon et al using player medical records for operative information and player surveys for follow-up. ${ }^{6,37}$ Menge et al included 60 NHL players undergoing 69 arthroscopy procedures, and Philippon et al included 28 NHL players undergoing 29 arthroscopy procedures. Intraoperative findings (all hips with labral tears) and RTP rates (both 100\%) were the same between the 2 studies. Philippon et al additionally reported on recovery time to return to skating and drills, demonstrating the benefit of player survey for short term follow-up data. Menge et al reported on postoperative career length and the factors correlated with it, demonstrating the benefit of internet-based data for long term follow-up career information. $^{6,37}$ No studies on FAI utilized the NHLISS or the AHMS as data sources.

\section{Hockey Groin Syndrome}

Brown et al, Irshad et al, Lacroix et al, and Jakoi et al investigated presentation and outcomes for players undergoing surgical repair of hockey groin syndrome, with Brown et al, Irshad et al, and Lacroix et al using player medical records as data sources and Jakoi et al using the internet. $^{7,38-40}$ The number of players included did not depend on the data source: Brown et al included 98 elite hockey players 67 of whom were NHL, Irshad et al included 22 NHL players, Lacroix et al included 11 NHL players, and Jakoi et al included 32 NHL players. Despite the different data sources, all reported similar RTP rates of 91-100\%. ${ }^{7,38-40}$ The use of player medical records allowed Brown et al, Irshad et al, and Lacroix et al to comment on surgical technique, intraoperative findings, and postoperative complications, which were similar across all three studies. ${ }^{38-40}$ In assessing postoperative performance, Jakoi et al investigated more parameters (games played, goals, assists, time on ice) than the other three studies which characterized performance outcomes based on the number of players returning to their preoperative level of competition and subjective reports - this suggests internet-based data may represent a more objective way of assessing postoperative performance. ${ }^{7}$ No studies on hockey groin syndrome utilized the NHLISS or the AHMS as data sources.

\section{ACL Tear}

Erickson et al, Mai et al, and Sikka et al investigated outcomes in NHL players following ACL reconstruction. $^{3,8,31}$ Erickson et al and Mai et al used internet-based sources while Sikka et al predominantly used the NHLISS (but also internet-based sources for performance statistics). All studies included similar numbers of players regardless of their data sources - Erickson et al included 36 NHL players, Mai et al included 350 ACL injuries of which 48 occurred in NHL players, and Sikka et al included 47 NHL players. However, Sikka et al were able to identify these players over a period of only 5 years while Erickson et al and Mai et al included players over a 13-year and 31-year range, respectively, demonstrating the value of the NHLISS in identifying large sample sizes for studies over a short period of time. Regardless of data source, all 3 studies reported similar RTP rates and recovery times $-89.4 \%$ to $97 \%$ RTP with recovery times ranging from 7.8 to 9.8 months. The 2 internet-based studies reported nearly identical postoperative career lengths of 4.5 years with no decrease in performance, while Sikka et al reported a postoperative career length of 2.8 years with a decrease in performance for forwards. $^{3,8,31}$ No studies on ACL tears utilized player medical records as data sources.

\section{Syndesmosis Sprain}

Both Mollon et al and Wright et al investigated presentation and outcomes of syndesmosis sprain in NHL players using the NHLISS to identify affected players and player medical records for more clinical data. ${ }^{27,29}$ Mollon et al included 105 players over 5 seasons from all teams while Wright et al included 19 players over 10 seasons from 2 teams. Mechanism of injury (external rotation), time of injury (during games rather than practices), and return to play rates $(100 \%)$ were similar between the 2 studies, though Wright et al reported a longer recovery time. 
Mollon et al additionally reported MRI findings in these players and Wright et al investigated treatments. ${ }^{27,29}$ These studies demonstrate how the NHLISS can be combined with player medical records to identify a wide spectrum of players but also analyze their specific medical data. No studies on syndesmosis sprains utilized internet-based sources as data sources.

\section{Discussion}

This systematic review summarizes all data published on orthopedic injuries sustained by professional ice hockey players competing in the NHL, characterizing all relevant publications based on anatomic injury site, data source, and level of evidence from January 1980 through March 2020. Our analysis of the reviewed literature found that NHL injury studies predominantly reported or focused on truncal and lower extremity injuries (71\%) compared to upper extremity injuries $(15 \%)$. These findings sharply contrast with findings reported by NFL injury studies which report fairly common occurrences of shoulder injuries, including AC separation, rotator cuff tears, and anterior instability, and which most often occurred during passing plays or resulted from direct trauma. ${ }^{9,48}$

The studies in our review consistently reported that orthopedic injuries in NHL players occurred more often during games than practices, a finding which has been reported in studies of college and international hockey players, as well as NFL players. ${ }^{48,49}$ Although orthopedic injuries in NHL players are usually associated with body-checking, incidental contact, and other collisions, the extensive and grueling nature of a full NHL season of 82 games plus playoffs may also contribute to player injury risk. ${ }^{13,25,26}$ Our review found that more than half of NHL players missed at least one game due to injury over the course of a season, and injuries were reported to be more common during the end of the regular season compared to the beginning of the regular season. The extensive and potentially sleep-depriving travel schedule of NHL players, which often involves multiple crosscontinental flights and back-to-back games in different time zones, may play a factor in the increasing injury risk over the progression of the season. Recently studied effects of disturbed circadian rhythms and sleep patterns on athletes' injury risk reflect a trend studied in other professional athletes with similarly demanding travel requirements, including NBA players and Italian professional soccer players. ${ }^{50-53}$ However, additional studies assessing the association of circadian regulation, sleep patterns, travel, and injury risk in NHL players need to be conducted before making direct conclusions.

Another potential cause of injury, fighting, is widely considered an integral part of playing ice hockey and even a tradition in the NHL, which is unique among US professional sports leagues in its policy refraining game officials from automatically ejecting players who are involved in fights. As a result, fighting poses injury risks unique to professional ice hockey players, especially when compared to professional athletes of other sports. However, injury data from NHL player fights has not been widely reported by studies. Only 2 studies reported fighting-related injuries in NHL players, with incidences ranging from $3.6 \%$ to $8 \%{ }^{13,23}$

Although there has been increasing adoption and use of injury surveillance systems across various sports leagues in recent years, few studies have utilized NHL injury surveillance databases which contain detailed and standardized player injury information. While NHL teams are "required to disclose that a player is expected to miss a game due to injury, or will not return to a game following an injury," they are not required to "disclose the specific nature of player injuries." 54 This is in contrast to the NFL and NBA, which require disclosure of the player's body part that is injured. ${ }^{55}$ Consequently, NHL injuries are typically reported as "upperbody injury," which includes concussions, and "lower-body injury," making it difficult for researchers to obtain usable information from these databases. ${ }^{55}$ By comparison, the vast majority of NFL studies of orthopedic injuries have used the NFL Injury Surveillance System. ${ }^{19}$ Although $38.5 \%$ of studies accessed player medical records, which offer the benefit of providing more detailed and standardized information about injuries compared to internet sources or media reports, a significant number of studies conducted NHL player surveys or surveys of team medical staff, including physicians and/or athletic trainers, comprising $33.3 \%$ and $69.2 \%$ of the total reviewed studies, respectively. However, survey data may be considered less reliable due to recall bias and variable nonresponse rates. In addition, publicly available data may provide limited information which are not as detailed or standardized as player medical records or injury surveillance databases, making it difficult to compare findings between internet-based studies. Given these findings, future projects looking to study NHL injuries may need to shift toward using data sources that contain more reliable and standardized information, such as the NHLISS or AHMS.

To our knowledge, this is the most up to date review of current literature on orthopedic injuries in the NHL; however, 
potential limitations remain. Only 39 studies were included in this review, some of which included very few NHL players. Furthermore, there was no standardized definition of injury that was used across all studies, introducing potential inaccuracy and limiting comparison between studies. The majority of studies $(89.7 \%$ ) provided lower-quality evidence (level III or IV). Additionally, a large number of studies in this review utilized internet-based searches of publicly available data (injury reports, press releases) and were not standardized, leaving open the potential for error. Furthermore, given the varied data reported by articles within this review, the authors have refrained from making definitive claims about player performance following injury, as this review focused on the epidemiology of orthopedic injuries in the NHL, and these comparisons are outside the scope of the study.

\section{Conclusion}

This systematic review provides NHL team physicians with a single source of the current literature regarding orthopedic injuries in NHL players. It is the first to comprehensively examine the wide range of injuries found in NHL players while providing a source of core knowledge - reflecting the current frequencies, outcomes, and risk factors - that can be used by treating physicians and medical staff for professional ice hockey teams. Furthermore, these findings can help guide effective injury prevention efforts through interventional assessments, and focused treatment.

\section{Disclosure}

Charles A Popkin, MD reports research and educational support from Arthrex and Gotham Surgical, outside the submitted work; and is a member of the USA Hockey Safety and Protective Equipment Committee. The authors report no other potential conflicts of interest for this work.

\section{References}

1. Popkin CA, Schulz BM, Park CN, Bottiglieri TS, Lynch TS. Evaluation, management and prevention of lower extremity youth ice hockey injuries. Open Access J Sports Med. 2016;7:167-176. doi:10.2147/OAJSM. S118595

2. Tuominen M, Stuart MJ, Aubry M, Kannus P, Parkkari J. Injuries in men's international ice hockey: a 7-year study of the International Ice Hockey Federation Adult World Championship Tournaments and Olympic Winter Games. Br J Sports Med. 2015;49(1):30-36.

3. Mai HT, Chun DS, Schneider AD, et al. Performance-based outcomes after anterior cruciate ligament reconstruction in professional athletes differ between sports. Am J Sports Med. 2017;45(10):2226-2232. doi: $10.1177 / 0363546517704834$

4. Mai HT, Chun DS, Schneider AD, Hecht AC, Maroon JC, Hsu WK. The difference in clinical outcomes after anterior cervical fusion, disk replacement, and foraminotomy in professional athletes. Clin Spine Surg. 2018;31(1):E80-E84. doi:10.1097/BSD.0000000000000570
5. Schallmo MS, Fitzpatrick TH, Yancey HB, Marquez-Lara A, Luo TD, Stubbs AJ. Return-to-play and performance outcomes of professional athletes in North America after hip arthroscopy from 1999 to 2016. Am J Sports Med. 2018;46(8):1959-1969.

6. Menge TJ, Briggs KK, Philippon MJ. Predictors of length of career after hip arthroscopy for femoroacetabular impingement in professional hockey players. Am J Sports Med.2016;44(9):2286-2291. doi:10.1177/ 0363546516650649

7. Jakoi A, O’Neill C, Damsgaard C, Fehring K, Tom J. Sports hernia in National Hockey League players: does surgery affect performance? Am J Sports Med. 2013;41(1):107-110. doi:10.1177/ 036354651

2462679

8. Erickson BJ, Harris JD, Cole BJ, et al. Performance and return to sport after anterior cruciate ligament reconstruction in national hockey league players. Orthop J Sports Med. 2014;2(9):232596711454883. doi:10.1177/2325967114548831

9. Christian RA, Lubbe RJ, Chun DS, Selley RS, Terry MA, Hsu WK. Prognosis following hip arthroscopy varies in professional athletes based on sport. Arthroscopy. 2019;35(3):837-842.e831. doi:10.1016/ j.arthro.2018.10.113

10. Yard EE, Collins CL, Comstock RD. A comparison of high school sports injury surveillance data reporting by certified athletic trainers and coaches. J Athl Train. 2009;44(6):645-652. doi:10.4085/10626050-44.6.645

11. Dick R, Agel J, Marshall SW. National collegiate athletic association injury surveillance system commentaries: introduction and methods. J Athl Train. 2007;42(2):173-182.

12. Goldberg AS, Moroz L, Smith A, Ganley T. Injury surveillance in young athletes: a clinician's guide to sports injury literature. Sports Med. 2007;37(3):265-278. doi:10.2165/00007256-200737030-00005

13. McKay CD, Tufts RJ, Shaffer B, Meeuwisse WH. The epidemiology of professional ice hockey injuries: a prospective report of six NHL seasons. Br J Sports Med. 2014;48(1):57-62. doi:10.1136/bjsports2013-092860

14. Baker HP, Varelas A, Shi K, Terry MA, Tjong VK. The NFL's chopblock rule change: does it prevent knee injuries in defensive players? Orthop J Sports Med. 2018;6(4):2325967118768446.

15. Ruestow PS, Duke TJ, Finley BL, Pierce JS. Effects of the NFL's amendments to the free kick rule on injuries during the 2010 and 2011 seasons. JOccupEnvironHyg. 2015;12(12):875-882. doi:10.1080/15459624.2015.1072632

16. Colello RJ, Colello IA, Abdelhameid D, Cresswell KG, Merchant R, Beckett E. Making football safer: assessing the current National Football League policy on the type of helmets allowed on the playing field. $J$ Neurotrauma. 2018;35 (11):1213-1223. doi:10.1089/neu.2017.5446

17. Yengo-Kahn AM, Johnson DJ, Zuckerman SL, Solomon GS. Concussions in the National Football League: a current concepts review. Am J Sports Med. 2016;44(3):801-811. doi:10.1177/ 0363546515580313

18. Decker WB, Baker AM, Ye X, Brown PJ, Stitzel JD, Gayzik FS. Development and multi-scale validation of a finite element football helmet model. Ann Biomed Eng. 2020;48(1):258-270. doi:10.1007/ s10439-019-02345-7

19. Kluczynski MA, Kelly WH, Lashomb WM, Bisson LJ, Systematic A. Review of the orthopaedic literature involving national football league players. Orthop J Sports Med. 2019;7(8):2325967119864356. doi:10.1177/2325967119864356

20. Moher D, Liberati A, Tetzlaff J, Altman DG, The PG. Preferred Reporting Items for Systematic Reviews and Meta-Analyses: The PRISMA Statement. PLoS Medicine. 2009;6(7):e1000097.

21. Tyler TF, Nicholas SJ, Campbell RJ, Donellan S, McHugh MP. The effectiveness of a preseason exercise program to prevent adductor muscle strains in professional ice hockey players. Am J Sports Med. 2002;30(5):680-683. doi:10.1177/03635465020300050801 
22. Tyler TF, Nicholas SJ, Campbell RJ, McHugh MP. The association of hip strength and flexibility with the incidence of adductor muscle strains in professional ice hockey players. Am J Sports Med. 2001;29 (2):124-128. doi:10.1177/03635465010290020301

23. Hutchinson MR, Milhouse C, Gapski M. Comparison of injury patterns in elite hockey players using ice versus in-line skates. Med Sci Sports Exerc. 1998;30(9):1371-1373.

24. Donaldson L, Li B, Cusimano MD. Economic burden of time lost due to injury in NHL hockey players. Inj Prev. 2014;20(5):347-349. doi:10.1136/injuryprev-2013-041016

25. Emery CA, Meeuwisse WH, Powell JW. Groin and abdominal strain injuries in the National Hockey League. Clin J Sport Med. 1999;9 (3):151-156. doi:10.1097/00042752-199907000-00006

26. Epstein DM, McHugh M, Yorio M, Neri B. Intra-articular hip injuries in national hockey league players: a descriptive epidemiological study. Am J Sports Med. 2013;41(2):343-348. doi:10.1177/ 0363546512467612

27. Mollon B, Wasserstein D, Murphy GM, White LM, Theodoropoulos J. High ankle sprains in professional ice hockey players: prognosis and correlation between magnetic resonance imaging patterns of injury and return to play. Orthop J Sports Med. 2019;7(9):232596711987157. doi:10.1177/2325967119871578

28. Lerebours F, Robertson W, Neri B, Schulz B, Youm T, Limpisvasti O. Prevalence of cam-type morphology in elite ice hockey players. $\mathrm{Am}$ J Sports Med. 2016;44(4):1024-1030. doi:10.1177/0363546515624671

29. Wright RW, Barile RJ, Surprenant DA, Matava MJ. Ankle syndesmosis sprains in National Hockey League players. Am J Sports Med. 2004;32(8):1941-1945.

30. Emery CA, Meeuwisse WH. Risk factors for groin injuries in hockey. Med Sci Sports Exerc. 2001;33(9):1423-1433. doi:10.1097/ 00005768-200109000-00002

31. Sikka R, Kurtenbach C, Steubs JT, Boyd JL, Nelson BJ. Anterior cruciate ligament injuries in professional hockey players. Am J Sports Med. 2016;44(2):378-383. doi:10.1177/0363546515616802

32. Watkins RG, Chang D, Watkins RG. Return to play after anterior cervical discectomy and fusion in professional athletes. Orthop J Sports Med. 2018;6(6):232596711877967. doi:10.1177/2325967118779672

33. Schroeder GD, McCarthy KJ, Micev AJ, Terry MA, Hsu WK. Performance-based outcomes after nonoperative treatment, discectomy, and/or fusion for a lumbar disc herniation in National Hockey League athletes. Am J Sports Med. 2013;41(11):2604-2608. doi:10.1177/ 0363546513499229

34. Dwyer T, Petrera M, Bleakney R, Theodoropoulos JS. Shoulder instability in ice hockey players. Incidence, mechanism, and MRI findings. Clin Sports Med. 2013;32(4):803-813. doi:10.1016/j. csm.2013.07.013

35. Rangavajjula A, Hyatt A, Raneses E, McCrossin J, Cohen S, DeLuca P. Return to play after treatment of shoulder labral tears in professional hockey players. Phys Sportsmed. 2016;44(2):119-125.

36. Larson CM, Ross JR, Kuhn AW, et al. Radiographic hip anatomy correlates with range of motion and symptoms in National Hockey League players. Am J Sports Med. 2017;45(7):1633-1639. doi:10.1177/ 0363546517692542

37. Philippon MJ, Weiss DR, Kuppersmith DA, Briggs KK, Hay CJ. Arthroscopic labral repair and treatment of femoroacetabular impingement in professional hockey players. Am J Sports Med. 2010;38 (1):99-104. doi:10.1177/0363546509346393

38. Brown RA, Mascia A, Kinnear DG, Lacroix V, Feldman L, Mulder DS. An 18-year review of sports groin injuries in the elite hockey player: clinical presentation, new diagnostic imaging, treatment, and results. Clin J Sport Med. 2008;18(3):221-226. doi:10.1097/JSM.0b013e318172831a

39. Irshad K, Feldman LS, Lavoie C, Lacroix VJ, Mulder DS, Brown RA. Operative management of "hockey groin syndrome": 12 years of experience in National Hockey League players. Surgery. 2001;130(4):759-766. doi:10.1067/msy.2001.118093
40. Lacroix VJ, Kinnear DG, Mulder DS, Brown RA. Lower abdomina pain syndrome in National Hockey League players: a report of 11 cases. Clin J Sport Med. 1998;8(1):5-9. doi:10.1097/00042752199801000-00002

41. Cooper DE, Conway JE. Distal semitendinosus ruptures in elite-level athletes: low success rates of nonoperative treatment. Am J Sports Med. 2010;38(6):1174-1178. doi:10.1177/0363546509361016

42. Sikka R, Fetzer G, Hunkele T, Sugarman E, Boyd J. Femur fractures in professional athletes: a case series. J Athl Train. 2015;50(4):442-448.

43. Erickson BJ, Harris JD, Fillingham YA, et al. Orthopedic practice patterns relating to anterior cruciate ligament reconstruction in elite athletes. Am J Orthop (Belle Mead NJ). 2015;44(12):E480-E485.

44. Schallmo MS, Singh SK, Barth KA, Freshman RD, Mai HT, Hsu WK. A cross-sport comparison of performance-based outcomes of professional athletes following primary microfracture of the knee. Knee. 2018;25(4):692-698. doi:10.1016/j.knee.2018.04.008

45. Simonet WT, Sim L. Boot-top tendon lacerations in ice hockey. J Trauma. 1995;38(1):30-31. doi:10.1097/00005373-199501000-00009

46. Baker JC, Hoover EG, Hillen TJ, Smith MV, Wright RW, Rubin DA. Subradiographic foot and ankle fractures and bone contusions detected by MRI in elite ice hockey players. Am J Sports Med. 2016;44(5):1317-1323. doi:10.1177/0363546515626181

47. Singh SK, Larkin KE, Kadakia AR, Hsu WK. Risk factors for reoperation and performance-based outcomes after operative fixation of foot fractures in the professional athlete: a cross-sport analysis. Sports Health. 2018;10(1):70-74. doi:10.1177/1941738117729660

48. Kaplan LD, Flanigan DC, Norwig J, Jost P, Bradley J. Prevalence and variance of shoulder injuries in elite collegiate football players. $\mathrm{Am}$ J Sports Med. 2005;33(8):1142-1146. doi:10.1177/0363546505274718

49. Anderson GR, Melugin HP, Stuart MJ. Epidemiology of injuries in ice hockey. Sports Health. 2019;11(6):514-519. doi:10.1177/ 1941738119849105

50. Burke TM, Lisman PJ, Maguire K, et al. Examination of sleep and injury among college football athletes. J Strength Cond Res. 2020;34 (3):609-616. doi:10.1519/JSC.0000000000003464

51. Copenhaver EA, Diamond AB. The value of sleep on athletic performance, injury, and recovery in the young athlete. Pediatr Ann. 2017;46(3):e106-e111. doi:10.3928/19382359-20170221-01

52. Huyghe T, Scanlan AT, Dalbo VJ, Calleja-Gonzalez J. The negative influence of air travel on health and performance in the national basketball association: a narrative review. Sports (Basel). 2018;6(3).

53. Lombardi G, Vitale JA, Logoluso S, et al. Circannual rhythm of plasmatic vitamin D levels and the association with markers of psychophysical stress in a cohort of Italian professional soccer players. Chronobiol Int. 2017;34(4):471-479. doi:10.1080/ 07420528.2017.1297820

54. Hackel S. The Morning Skate: N.H.L.'s New Injury-Disclosure Policy Draws Heavy Criticism. Slapshot Blog, New York Times; 2008.

55. Deubert CRLH, Cohen IG. Comparing Health-Related Policies \& Practices in Sports: The NFL and Other Professional Leagues. Boston, MA: Harvard Law School; 2017.

56. Kantrowitz DE, Padaki AS, Ahmad CS, Lynch TS. Defining platelet-rich plasma usage by team physicians in elite athletes. Orthop J Sports Med. 2018;6(4):232596711876707. doi:10.1177/ 2325967118767077

57. Rowan CP, Kuropkat C, Gumieniak RJ, Gledhill N, Jamnik VK. Integration of the functional movement screen into the National Hockey League Combine. J Strength Cond Res. 2015;29 (5):1163-1171. doi:10.1519/JSC.0000000000000757

58. Van Pelt KL, Lapointe AP, Galdys MC, Dougherty LA, Buckley TA, Broglio SP. Evaluating performance of National Hockey League players after a concussion versus lower body injury. J Athl Train. 2019;54(5):534-540. doi:10.4085/1062-6050-218-18 


\section{Publish your work in this journal}

Open Access Journal of Sports Medicine is an international, peerreviewed, open access journal publishing original research, reports, reviews and commentaries on all areas of sports medicine. The

manuscript management system is completely online and includes a very quick and fair peer-review system. Visit http://www.dovepress. com/testimonials.php to read real quotes from published authors.

Submit your manuscript here: http://www.dovepress.com/open-access-journal-of-sports-medicine-journal 